\title{
Solution to blue garlic challenge
}

\section{Hervé This}

(C) Springer-Verlag Berlin Heidelberg 2014

The winner of the blue garlic challenge (published in volume 406 issue 1) is:

Wai-Yin Lau, Department of Chemistry, University of Hong Kong, Hong Kong, China

The award entitles the winner to select a Springer book of her choice up to a value of $€ 100$.

Our Congratulations!

\section{Solution}

The explanation of the "greening" or "blueing" of garlic (Allium sativum L.) and "pinking" of onion (Allium cepa L.) given here will conclude with a new challenge of its own.

Color changes of Allium tissues have been studied by several investigators since Joslyn, in 1958 [1]. In the 1960s, the reactions involved in the formation of pink pigment in onion purée were investigated [2] and a three-step reaction scheme was proposed: (1) the formation of a colorless, ethersoluble substance [the color developer (CD)] by the catalytic action of alliinase (enzyme EC 4.4.1.4) upon then-unknown precursors in the fraction of neutral and basic amino acids; (2) the formation of a colorless, ether-insoluble pigment precursor (PP) from the color developer and an amino acid such as glycine; (3) the formation of a pink pigment from the pigment precursor PP and a naturally occurring carbonyl (NOC) substance such as formaldehyde. It was then assumed [3] that a blue pigment-forming "carbonyl" was present in garlic, and when reacted with the pigment precursor PP in place of NOC from onion, a blue color would form.

This article is the solution to the Analytical Challenge to be found at http://dx.doi.org/10.1007/s00216-013-7464-2

\footnotetext{
H. This $(\bowtie)$

INRA/AgroParisTech, UMR 1145, Group of Molecular Gastronomy, 16 rue Claude Bernard, 75005 Paris, France

e-mail: herve.this@paris.inra.fr
}

Subsequently, it was found [4] that the blue pigment-forming "carbonyl" was derived from isoalliin (a substrate for alliinase, a minor precursor in garlic flavor, but the major precursor of onion flavor), and the same reactions were responsible for "greening" of garlic and of the mixture of garlic and onion.

But our understanding of the Allium chemistry advanced significantly when Eric Block and his colleagues [5-8] introduced methods for the study of Allium chemistry and produced a wealth of results based on such methods. Based on their work, it was established (see Fig. 1) that the pigment precursor was a 3,4-dimethylpyrrole derivative. It is thought to be formed by condensation of the amine group of Allium amino acids with the thial/thial S-oxide formed by [3]sigmatropic rearrangement of bis-1-propenyl thiosulfinate, in turn formed by the action of alliinase on 1-PeCSO (isoalliin). The thial/thial S-oxide is an intermediate in the formation of zwiebelanes, and is closely related in structure to (Z,Z)-d,12,3-dimethyl-1,4-butanedithial S,S'-dioxide, a compound isolated from onion preparations, which could play a role in forming the pigment precursor. A second key aspect to the formation of colors in Allium preparation requires the intermediacy of thioacrolein.

Using such information, a positive correlation between thiosulfinate concentration and pink pigment formation was first observed [9], and 1-propenyl-containing thiosulfinates were confirmed to be the major color-developing compounds [10]. Then in 2005, Bai et al. [11] studied the mechanisms of the green color formation in "Laba" garlic, a preserve of garlic including vinegar and sometimes sugar: both alliinase and acetic acid are required for the color formation, and the decrease in the total thiosulfinates in garlic cloves is associated with the pigment formation.

The plant chemistry, however, is always wonderfully complex, so that other studies are not useless. Imai et al. [12] established a model reaction system that comprised only welldefined constituents and reported identifications of new 
Fig. 1 Formation of various visible light absorbing compounds from Allium bulbs (from [15])

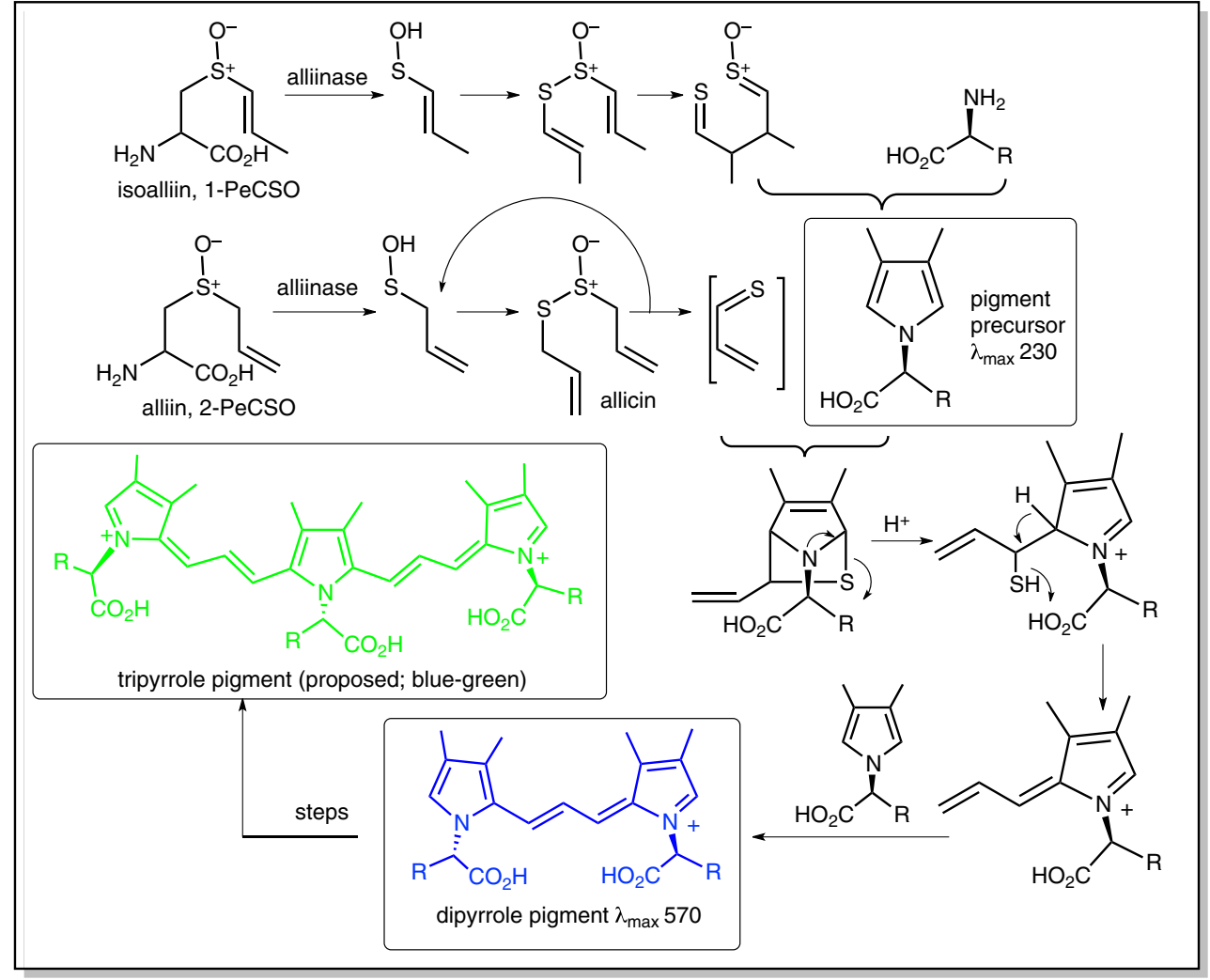

substances, which were involved in the pigment formation along with various conditions that affected color development. Addition of glycine suggested that proteins in the garlic juice participated presumably in the pigment formation and that they were less reactive than $75 \% \mathrm{MeOH}$-soluble free amino acids, such as glycine. The active compound that yielded blue color when combined with unheated onion juice was isolated from unheated garlic juice and was confirmed to be allicin, which derived from alliin by the action of alliinase. It was also confirmed that a vivid-blue color could be produced by using a highly defined model reaction system comprising only isolated alliin, pure glycine, and purified garlic alliinase. Later, Imai et al. $[13,14]$ isolated two pigment precursors and a reddish-purple pigment (PUR-1) and determined their chemical structures.

In 2007, Lee et al. [15] purified the green pigment, responsible for greening in crushed garlic cloves, and they analyzed it by liquid chromatography-electrospray ionization mass spectrometry (LC-ESI-MS), fast atom bombardment mass spectrometry (FAB-MS), matrix-assisted laser desorption/ ionization time-of-flight mass spectrometry (MALDI-TOFMS), and nuclear magnetic resonance (NMR) spectroscopy. The purified green pigment was highly polar and slightly viscous, with a garlic odor, and easily turned to a yellow or brown color with exposure to room temperature. The absorption spectrum in methanol showed a crude methanolic green pigment-like profile with two absorbance maxima at 440 and
$590 \mathrm{~nm}$. Although complete isolation of the 411 Da compound for proper structure elucidation was not achieved in their experiments, the MS and NMR spectra of the $411 \mathrm{Da}$ green pigment suggested the ambiguous structural assignment of one sulfur atom and odd number of nitrogen atoms, with 25-30 carbon atoms, including aromatic ring. Therefore, it was envisioned that the green pigment observed in crushed garlic cloves was a new sulfur-containing nitrogenous watersoluble compound differing significantly from all previously reported green pigments in plants.

Obviously, the story is not fully over, but the worst is when one tries to get this color, as I did. When I simply put peeled garlic cloves in vinegar, the color did not appear, whether I boiled the system or not, and even after 2 wk of maceration. According to Block [15], garlic heads should be aged before immersion in vinegar in order for the color to appear. Let's meet again in 4 months from now!

\section{References}

1. Joslyn MA, Peterson RG (1958) Food discoloration, reddening of white onion bulb purees. J Agric Food Chem 6:754-765

2. Shannon S, Yamaguchi M, Howard FD (1967) Reactions involved in formation of a pink pigment in onion purees. J Agric Food Chem 15: $417-422$ 
3. Yamaguchi M, Shannon S, Howard FD, Joslyn MA (1965) Factors affecting the formation of a pink pigment in purees of onion. Proc Am Soc Hortic Sci 86:475-483

4. Shannon S, Yamaguchi M, Howard FD (1967) Precursors involved in the formation of pink pigments in onion purees. J Agric Food Chem 15:423-426

5. Bayer T, Wagner H, Block E, Grisoni S, Zhao SH, Neszmelyi A (1989) Zwiebelanes: Novel 2,3-dimethyl-5,6-dithibicyclo[2.1.1]hexanes from onion. J Am Chem Soc 111:3085-3086

6. Block E, Bayer T (1990) (Z,Z)-d,1-2,3-Dimethyl-1,4-butanedithial S, S'-dioxide: A novel biologically active organosulfur compound from onion. Formation of vic-disulfoxide in onion extracts. J Am Chem Soc 112:4584-4585

7. Block E (1992) The organosulfur chemistry of the genus Allium implications for organic sulfur chemistry. Angew Chem Int Edn 31: $1135-1178$

8. Block E, Bayer T, Naganathan S, Zhao SH (1996) Allium chemistry: Synthesis and sigmatropic rearrangements of alk(en)yl 1-propenyl disulfide S-oxides from cut onion and garlic. J Am Chem Soc 118: 2799-2810

9. Lukes TM (1986) Factors governing the greening of garlic puree. J Food Sci 51:1577-1582
10. Lee CH, Parkin KL (1998) Relationship between thiosulfinates and pink discoloration in onion extracts, as influenced by $\mathrm{pH}$. Food Chem 61:345-350

11. Bai B, Chen F, Wang Z, Liao X, Zhao G, Hu X (2006) Mechanism of the greening color formation of "Laba" garlic, traditional homemade Chinese food product. J. Agric. Food Chem. 2005, 53, 7103-7107. Imai S.; Akita K.; Tomotake M.; Sawada, H.; Identification of Two Novel Pigment Precursors and a Reddish-Purple Pigment Involved in the Blue-Green Discoloration of Onion and Garlic. J Agric Food Chem 54:843-847

12. Imai $\mathrm{S}$, Tsuge $\mathrm{N}$, Tomotake $\mathrm{M}$, Nagatome $\mathrm{Y}$, Sawada H, Nagata $\mathrm{T}$, Kumagai H (2002) An onion enzyme that makes the eyes water. Nature 419:685

13. Imai S, Akita K (2006) Tomotake, M; Shinsuke Imai, Kaori Akita, Muneaki Tomotake, Hiroshi Sawada, Model Studies on Precursor System Generating Blue Pigment in Onion and Garlic. J Agric Food Chem 54:848-852

14. Lee EJ, Cho JE, Kim JH, Lee SK (2007) Green pigment in crushed garlic (Allium sativum L.) cloves: Purification and partial characterization. Food Chem 101:1677-1686

15. Block E (2009) Garlic and other Alliums: The Lore and the Science. Royal Society of Chemistry, London 